\title{
67/w mit hochgradiger Hemiparese rechts und 2-maligem generalisiertem Krampfanfall
}

\author{
Vorbereitung auf die Facharztprüfung: Fall 47
}

\section{Prüfungssimulation}

\section{Fallschilderung für den Prüfungskandidaten}

Eine 67-jährige Patientin wurde als Notfall mit einer hochgradigen rechtsseitigen Hemiparese, Aphasie mit Verdacht auf einen Schlaganfall aus einem anderen Haus übernommen, bei der Aufnahme fiel eine Temperaturerhöhung auf. Die Vorstellung in der vorherigen Klinik erfolgte aufgrund einer zunächst passageren Sprachstörung. Eine im Vorkrankenhaus erfolgte Bildgebung (cCT (craniale Computer-Tomographie) und CTA (computertomographische Angiographie)) war unauffällig.

Nach Rückbildung der passageren Sprachstörung hatte die Patientin im Vorkrankenhaus eine hochgradige Hemiparese rechts und 2 generalisierte Krampfanfälle mit einer Blickwendung nach rechts entwickelt, daraufhin erfolgte die Verlegung in unser Krankenhaus.

\section{Klinische Untersuchung}

Wache, fieberhafte $\left(39,5^{\circ} \mathrm{C}\right)$, global aphasische Patientin, ohne Meningismuszeichen, die Aufforderungen nicht folgen kann. Hochgradige armbetonte spastische Hemiparese rechts mit beidseits positiven Pyramidenbahnzeichen (positiver Babinski-Reflex beidseitig).

In der Lumbalpunktion lymphomonozytäres Zellbild mit 28 Zellen. Die Liquorchemie wies ein Gesamtprotein von $72 \mathrm{mg} / \mathrm{dl}$, einen Laktatwert von 22,3 mg/dl, und eine Liquorglukosekonzentration von $46 \mathrm{mg} / \mathrm{dl}$ auf.

Elektroenzephalographisch zeigte sich ein rhythmischer Burst von Komplexen von „sharp waves“ und „spike waves“.

In der Magnetresonanztomographie (MRT) zeigte sich links frontotemporal unter Einbeziehung von Temporallappen, Operculum, der Inselrinde sowie des linken Thalamus eine deutliche Ödembildung (Abb. 1 a-d).

\section{Redaktion}

P. Berlit, Berlin

\section{? Prüfungsfragen}

- Was vermuten Sie und warum?

- Welche therapeutischen Maßnahmen sollten unverzüglich eingeleitet werden?

- Ist eine weiterführende Diagnostik indiziert?

- Wie ist die Prognose einzuschätzen? Welche Komplikationen können auftreten?

- Nennen Sie wichtige Differenzialdiagnosen.

- Wann kann eine bei Verdacht eingeleitete Therapie mit Aciclovir beendet werden? 


\section{Antworten}

Was vermuten Sie und warum?

- Anhand der Befunde von Liquordiagnostik sowie der bildgebenden Befunde ist an eine Herpes-simplex-Virus-Enzephalitis (HSVE) zu denken.

- Typisch sind die geringe lymphozytäre Pleozytose und die asymmetrischen entzündlich-hämorrhagischen Läsionen in Temporallappen und limbischem System.

- Bei einer Herpes-simplex-Virus-Enzephalitis (HSVE) kommt es in ungefähr $32 \%$ zu Krampfanfällen, fakultativ können auch neurologische Ausfälle, wie im obigen Fall beschrieben, auftreten.

\section{? Welche therapeutischen Maßnahmen sollten unverzüg-} lich eingeleitet werden?

- Bereits bei Verdacht auf eine Herpes-simplex-Virus-Enzephalitis muss unverzüglich eine Therapie mit Aciclovir eingeleitet werden.

- Die Gabe von Aciclovir erfolgt in einer Dosis von 10 mg/kgKG alle 8 h i.v. über 14-21 Tage.

Cave. Wegen der Nephrotoxizität sind eine langsame Infusionsgeschwindigkeit und ausreichende Hydrierung wichtig. Bei Niereninsuffizienz ist eine Dosisreduktion erforderlich.

- In Anbetracht der 2-maligen Krampfanfälle sowie des EEGBefundes sollte eine antikonvulsive Therapie, z. B. mit Levetiracetam, erfolgen.

\section{? Ist eine weiterführende Diagnostik indiziert?}

- Ja, eine weiterführende Diagnostik ist indiziert. Beim klinischen Verdacht auf eine HSVE muss immer eine Liquordiagnostik mit HSV-PCR und Antikörperbestimmung erfolgen.

- Eine HSV-spezifische PCR aus dem Liquor bestätigte die HSVE. Die HSV-PCR hat eine Sensitivität von $>95 \%$ und eine Spezifität vom $100 \%$ und kann die Diagnose frühzeitig sichern.
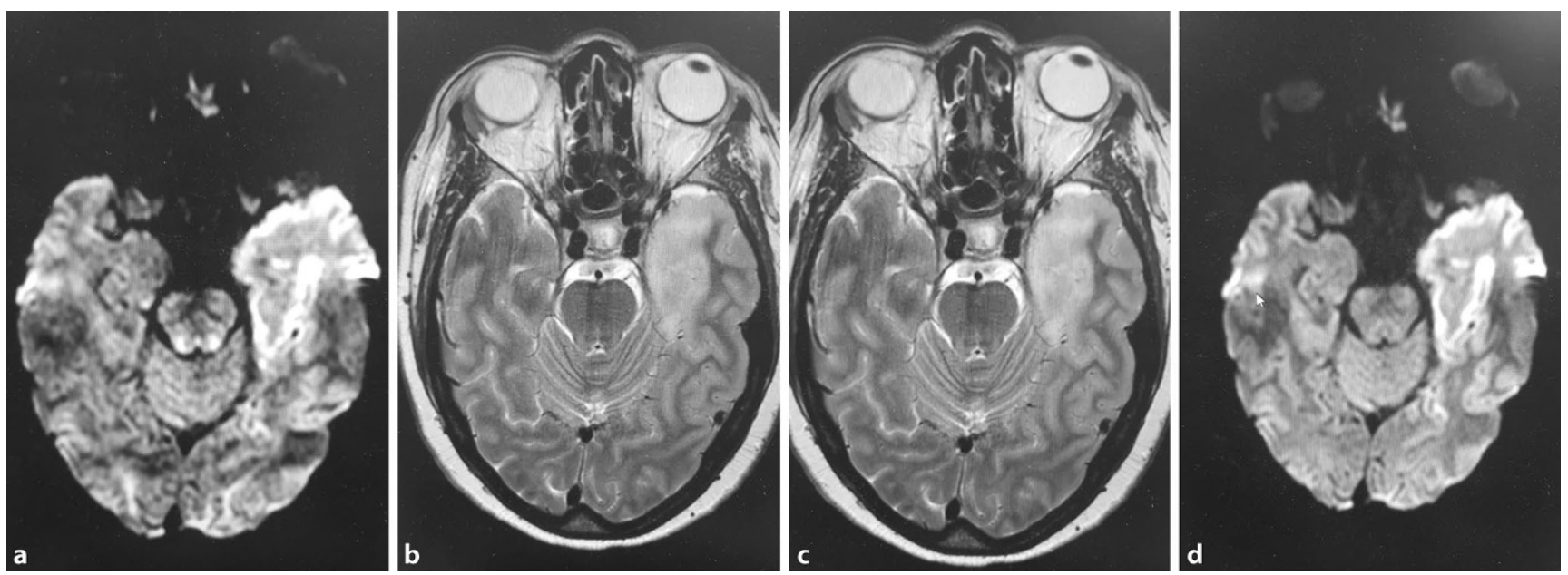

Abb. 1 Magnetresonanztomographie: es zeigte sich links frontotemporal unter Einbeziehung von Temporallappen, Operculum, der Inselrinde sowie des linken Thalamus eine deutliche Ödembildung 
- Hirndrucksteigerungen können u. U. eine Hemikraniektomie erfordern.

Cave. Bei einem Wiederauftreten von Symptomen nach Besserung ist an eine sekundäre Autoimmunenzephalitis zu denken! Es sind dann NMDA-R-Antikörper nachzuweisen.

- Aciclovir kann nephrotoxisch wirken und ein akutes Nierenversagen verursachen; daher muss die Nierenfunktion überwacht werden (Dosisanpassung!). In seltenen Fällen kommt es zur Aciclovirunverträglichkeit mit der Entwicklung von Psychosen.

\section{? Nennen Sie wichtige Differenzialdiagnosen}

- Insbesondere bei älteren Patienten mit Vorerkrankungen kann ein durch Infekt oder medikamentös ausgelöstes Delir zu ähnlichen Symptomen führen.

- Enzephalitiden durch andere Viren (z. B. VZV, SARS-CoV-2) oder Bakterien (Tb)

- Autoimmunenzephalitiden

- Vaskulitiden, z. B. Granulomatose mit Polyangiitis, PACNS

- Gliome oder Lymphome des Temporallappens

- Akute disseminierte Enzephalomyelitis (ADEM)

- Progressive multifokale Leukenzephalopathie (PML)

? Wann kann eine bei Verdacht eingeleitete Therapie mit Aciclovir beendet werden?

- Wenn der klinische Verlauf nicht zur Diagnose einer HSVE passt, z. B. wacher, orientierter Patient ohne neuropsychiatrische Symptome (Differenzialdiagnose: postiktales Bild bei Temporallappenepilepsie)

- Wenn Liquorbefund und MRT über mehr als 4 Tage nach Symptombeginn unauffällig bleiben. Eine Kontrollpunktion und wiederholte PCR und Antikörperindexbestimmung sind grundsätzlich indiziert.

- Wenn die Untersuchungen eine andere Ursache der Symptomatik belegen.

Weiterführende Literatur

1. Meyding-LamadéU et al (2018) Virale Meningoenzephalitis, S1-Leitlinie. Leitlinien für Diagnostik und Therapie in der Neurologie. Deutsche Gesellschaft für Neurologie, Berlin (Online: www.dgn.org/leitlinien)

2. Meyding-Lamadé U, Jacobi C, Martinez-Torres Fet al (2019) The German trial on Aciclovir and Corticosteroids in Herpes-simplex-virus-Encephalitis (GACHE): a multicenter, randomized, double-blind, placebo-controlled trial. Neurol Res Pract 1:26. https://doi.org/10.1186/s42466-019-0031-3

3. Raschilas F, WolffM, Delatour F, Chaffaut C, De Broucker T, Chevret S et al (2002)

Outcome of and prognostic factors for herpes simplex encephalitis in adult patients: results of a multicenter study. Clin Infect Dis 35:254-260

4. Saraya AW, Wacharapluesadee S, Petcharat Set al (2016) Normocellular CSF in herpes simplex encephalitis. BMC Res Notes 9:95

5. Thakur KT, Motta M, Asemota AO, Kirsch HL, Benavides DR, Schneider EB et al (2013) Predictors of outcome in acute encephalitis. Neurology 81:793-800
Korrespondenzadresse

Prof. Dr. med. U. Meyding-Lamadé

Klinik für Neurologie,

Krankenhaus Nordwest GmbH

Steinbacher Hohl 2-26, 60488 Frankfurt, Deutschland

meyding-lamade.uta@khnw.de

Schlüsselwörter. Herpes-simplex-Virus · Epilepsie · Krampfanfall · Aphasie · Autoimmunenzephalitis · Enzephalitis

\section{Einhaltung ethischer Richtlinien}

Interessenkonflikt. U. Meyding-Lamadé und E. M. Craemer geben an, dass kein Interessenkonflikt besteht.

Für diesen Beitrag wurden von den Autoren keine Studien an Menschen oder Tieren durchgeführt. Für die aufgeführten Studien gelten die jeweils dort angegebenen ethischen Richtlinien. Für Bildmaterial oder anderweitige Angaben innerhalb des Manuskripts, über die Patienten zu identifizieren sind, liegt von ihnen und/oder ihren gesetzlichen Vertretern eine schriftliche Einwilligung vor.

The supplement containing this article is not sponsored by industry. 